\title{
A perturbative approach to the polaron shift of excitons in transition metal dichalcogeniedes
}

\author{
J. C. G. Henriques ${ }^{1,2}$ and N. M. R. Peres ${ }^{1,2}$ \\ ${ }^{1}$ Department and Centre of Physics, and QuantaLab, \\ University of Minho, Campus of Gualtar, 4710-057, Braga, Portugal and \\ ${ }^{2}$ International Iberian Nanotechnology Laboratory (INL), \\ Av. Mestre José Veiga, 4715-330, Braga, Portugal
}

\begin{abstract}
In this paper we study the phonon's effect on the position of the 1s excitonic resonance of the fundamental absorption transition line in two-dimensional transition metal dichalcogenides. We apply our theory to $\mathrm{WS}_{2}$ a two-dimensional material where the shift in absorption peak position has been measured as a function of temperature. The theory is composed of two ingredients only: i) the effect of longitudinal optical phonons on the absorption peak position, which we describe with second order perturbation theory; ii) the effect of phonons on the value of the single particle energy gap, which we describe with the Huang Rhys model. Our results show an excellent agreement with the experimentally measured shift of the absorption peak with the temperature.
\end{abstract}

One of the most prominent and studied types of twodimensional materials are transition metal dichalcogeniedes (TMDs) [1. These, often semi-conducting, materials present remarkable electronic and optical properties, intrinsically related with their excitonic response. An exciton is a quasi-particle corresponding to a bound electron-hole pair interacting via a Coulomb-like potential. Due to the reduced dielectric screening in twodimensional materials, these quasi-particles are more tightly bound, and thus more stable, than their threedimensional analogues. Phonons, the quanta of atomic vibrational energy, are known to have a significant impact on the optical properties of TMDs, especially due to their interaction with excitons 2 25. The exciton-phonon coupling influences both the line width and the peak position of the different absorption resonances associated with the optically active excitonic states in TMDs. Indeed, this effect has been reported recently in Ref. [6], where it was shown that the $1 s$ excitonic peak was red-shifted as the temperature increased, accompanied by a concomitant increased line width of the resonance. The coupling of phonons to excitons also affects their radiative lifetime, and allows the access to optically dark states via intervalley scattering channels [7]. Studies on the temperature dependence of the optical properties of these materials are highly relevant to accurately predict their applicability in different technological applications which are required to work at room temperature.

The problem of electron-phonon interaction is by no means a simple one, giving rise to, for example, phononmediated supercondutivity and the polaron problem, an electron dressed with a cloud of phonons. While the former problem can be dealt with an approximate canonical transformation, the latter one is, in general, nonperturbative. However, to address the effect of phonon's on the position peak of the absorption resonance a perturbative approach, up to seconder in the electronphonon interaction, suffices. However, as derived from traditional perturbation theory, we end up with a sum over all states of the non-interacting problem, whose ef- fective summation is out of reach simply because it requires all the eigenstates of the non-interacting system, which may not be known. Even in the cases where they are known, the integrals are of insurmountable difficulty. Therefore, we follow a different path for circumventing the sum over states. We use the Dalgarno-Lewis approach [8] which shifts the sum over states problem to the solution of an non-homogeneous differential equation. In this procedure, only one eigenstate of the unperturbed theory is required together with the solution of the aforementioned differential equation.

In this paper we consider a two dimensionalTMD whose electrons and holes, interacting via a Coulomblike potential, may give rise to exciton formation. In order to study the effect of temperature on the excitonic properties, we use a similar model to the one employed in Ref. [9, where a Frohlich-like Hamiltonian [10] was used to characterize the interaction of optical phonons with electrons and holes in polar crystals. Contrary to Ref. [9], where 3D systems were considered, we will focus on excitons on 2D materials, leading to a difference in the form of the interaction term [11. Moreover, contrary to the aforementioned work where only the case of $T=0 \mathrm{~K}$ was considered, our calculations cover any temperature value. The Hamiltonian of the considered system in the center of mass frame of the electron-hole pair reads:

$$
\mathcal{H}=H_{0}+H_{1}+H_{2}+H_{3}+H_{4}
$$

where

$$
\begin{aligned}
& H_{0}=\frac{p^{2}}{2 \mu}+V(r), \quad H_{1}=\sum_{\mathbf{q}} \hbar \omega_{\mathbf{q}} a_{\mathbf{q}}^{\dagger} a_{\mathbf{q}} \\
& H_{2}=-\frac{U}{A^{1 / 2}} \sum_{\mathbf{q}} \frac{i}{\sqrt{q}} a_{\mathbf{q}} e^{i \mathbf{q} \cdot\left(m_{h} / M\right) \mathbf{r}}+h . c . \\
& H_{3}=\frac{U}{A^{1 / 2}} \sum_{\mathbf{q}} \frac{i}{\sqrt{q}} a_{\mathbf{q}} e^{-i \mathbf{q} \cdot\left(m_{e} / M\right) \mathbf{r}}+h . c . \\
& H_{4}=\frac{1}{2 M}\left(\mathbf{K}-\sum_{\mathbf{q}} \hbar \mathbf{q} a_{\mathbf{q}}^{\dagger} a_{\mathbf{q}}\right)^{2} .
\end{aligned}
$$


The term $H_{0}$ is the Hamiltonian of the exciton, with $m_{e / h}$ the electron/hole effective mass, $\mu^{-1}=m_{e}^{-1}+m_{h}^{-1}$ the reduced mass of the electron-hole pair, $p$ their relative momentum, $\mathbf{r}$ their relative position vector and $V(r)$ the electron-hole interaction potential which we model using the Rytova-Keldysh potential [12, 13]

$$
V(r)=-\frac{e^{2}}{4 \pi \epsilon_{0}} \frac{\pi}{2 r_{0}}\left[\mathbf{H}_{0}\left(\frac{\kappa r}{r_{0}}\right)-Y_{0}\left(\frac{\kappa r}{r_{0}}\right)\right]
$$

where $e$ is the elementary charge, $\epsilon_{0}$ is the vacuum permittivity, $\kappa$ is the mean dielectric constant of the media above and below the TMD monolayer, $r_{0}$ is a materialdependent screening length (which is microscopically related to the material's polarizability), $\mathbf{H}_{0}$ is the Struve function of the first kind and $Y_{0}$ the Bessel function of the second kind, both of order zero. In the total Hamiltonian, the term $H_{1}$ describes the thermally excited phonons and $a_{\mathbf{q}}^{\dagger} / a_{\mathbf{q}}$ refers to the creation/annihilation operator of a phonon with momentum $\mathbf{q}$ and energy $\hbar \omega_{\mathbf{q}}$. In this term we only consider the contribution originating from longitudinal-optical (LO) phonons. Also, we will consider that the energy $\hbar \omega_{\mathbf{q}}$ is independent of momentum and equal to a constant value $\hbar \omega_{\mathrm{LO}}$ when numerical results are computed. The terms $H_{2}$ and $H_{3}$ correspond to the interaction between the phonons and the electrons and holes, $A$ is the area of the 2D monolayer, $M=m_{e}+m_{h}$ and $U$ is the coupling potential defined as [11]

$$
U=\hbar \omega_{\mathrm{LO}}(\sqrt{2} \pi \alpha)^{1 / 2}\left(\frac{\hbar}{m_{0} \omega_{\mathrm{LO}}}\right)^{1 / 4}
$$

with $m_{0}$ the bare electron mass, $\alpha$ a dimensional coupling constant, which we will consider as a fitting parameter, but whose typical value is between 2-5 [11. Finally, the term $H_{4}$ depends on the center of mass momentum $\mathbf{K}$. It is not in general expected that a term which depends on the center of mass momentum will play a significant role in the system's internal dynamics, thus hereinafter, just like in Ref. 9], we neglect its contribution to $\mathcal{H}$, that is

$$
\mathcal{H} \approx H_{0}+H_{1}+H_{2}+H_{3} .
$$

Now, in order to compute the effects of the coupling of the excitons to the LO phonons we will follow a perturbative approach, taking $H_{0}+H_{1}$ as the unperturbed Hamiltonian and $\mathrm{H}_{2}+\mathrm{H}_{3}$ as the perturbative term. From second order perturbation theory, we write the energy correction to the system's ground state as

$$
\Delta E=\sum_{\nu_{X} \nu_{p h}} \frac{\left|\left\langle 1 s ; n_{p h}(T)\left|H_{2}+H_{3}\right| \nu_{X} ; \nu_{p h}\right\rangle\right|^{2}}{E_{G S}-E_{\nu_{X} \nu_{p h}}},
$$

where $\nu_{X}$ and $\nu_{p h}$ refer to the states of the exciton and the phonons, respectively, with a combined energy $E_{\nu_{X} \nu_{p h}}, 1 s$ refers to the most tightly bound excitonic state and $n_{p h}(T)$ corresponds to the phonon distribution at a temperature $T$, with a combined energy $E_{G S}$. The sum runs over all the $\nu_{X}$ and $\nu_{p h}$ except $\left\{\nu_{X}, \nu_{p h}\right\}=$ $\left\{1 s, n_{p h}(T)\right\}$. A direct evaluation of Eq. (5) would undoubtedly be a daunting task, with little probability of success, since all the excitonic wave functions would be required and an infinite number of matrix elements would have to be evaluated. As an alternative route, one can follow the Dalgarno-Lewis approach [8] in order to bypass the sum over states. This ingenious approach consists on the introduction of an operator, defined through a differential equation, which when inserted in Eq. (5) allows the sum over states to be removed. The problem of computing $\Delta E$ is then reduced to the evaluation of a single matrix element. More specifically, it is possible to show that Eq. (5) can be written as the sum of four contributions

$$
\Delta E=\Delta E_{1}+\Delta E_{2}+\Delta E_{3}+\Delta E_{4},
$$

where

$$
\begin{aligned}
\Delta E_{1}=\frac{U^{2}}{A} \sum_{\nu_{X}} \sum_{\mathbf{q}} \frac{1}{q}\left\{\frac{n_{p h}(\mathbf{q}, T)+1}{E_{1 s}-E_{\nu_{X}}-\hbar \omega_{\mathbf{q}}}\left\langle 1 s\left|e^{-i \mathbf{q} \cdot\left(m_{e} / M\right) \mathbf{r}}\right| \nu_{X}\right\rangle\left\langle\nu_{X}\left|e^{i \mathbf{q} \cdot\left(m_{e} / M\right) \mathbf{r}}\right| 1 s\right\rangle\right. \\
\left.+\frac{n_{p h}(\mathbf{q}, T)}{E_{1 s}-E_{\nu_{X}}+\hbar \omega_{\mathbf{q}}}\left\langle 1 s\left|e^{i \mathbf{q} \cdot\left(m_{e} / M\right) \mathbf{r}}\right| \nu_{X}\right\rangle\left\langle\nu_{X}\left|e^{-i \mathbf{q} \cdot\left(m_{e} / M\right) \mathbf{r}}\right| 1 s\right\rangle\right\}
\end{aligned}
$$

and

$$
\begin{aligned}
\Delta E_{3}=-\frac{U^{2}}{A} \sum_{\nu_{X}} \sum_{\mathbf{q}} \frac{1}{q} & \left\{\frac{n_{p h}(\mathbf{q}, T)+1}{E_{1 s}-E_{\nu_{X}}-\hbar \omega_{\mathbf{q}}}\left\langle 1 s\left|e^{-i \mathbf{q} \cdot\left(m_{e} / M\right) \mathbf{r}}\right| \nu_{X}\right\rangle\left\langle\nu_{X}\left|e^{-i \mathbf{q} \cdot\left(m_{h} / M\right) \mathbf{r}}\right| 1 s\right\rangle\right. \\
& \left.+\frac{n_{p h}(\mathbf{q}, T)}{E_{1 s}-E_{\nu_{X}}+\hbar \omega_{\mathbf{q}}}\left\langle 1 s\left|e^{-i \mathbf{q} \cdot\left(m_{h} / M\right) \mathbf{r}}\right| \nu_{X}\right\rangle\left\langle\nu_{X}\left|e^{-i \mathbf{q} \cdot\left(m_{e} / M\right) \mathbf{r}}\right| 1 s\right\rangle\right\},
\end{aligned}
$$

with $E_{1 s}$ and $E_{\nu_{X}}$ the energies of the $1 s$ and $\nu_{X}$ excitonic

states, respectively, and $n_{p h}(\mathbf{q}, T)$ the Bose-Einstein dis- 
tribution function for phonons with energy $\omega_{\mathbf{q}}$ at a temperature $T$. Here we still consider the phonon energy as a function of the momentum in order to present general expressions, but later we will consider $\omega_{\mathbf{q}}=\omega_{\mathrm{LO}}$ when numerical results are computed. The expressions for $\Delta E_{2}$ and $\Delta E_{4}$ follow directly from these two by replacing $m_{e}\left(m_{h}\right)$ with $m_{h}\left(m_{e}\right)$. Each of the four contributions is made up of two terms with distinct physical origin: one originating from phonon emission and the other from phonon absorption. In the limit of vanishing temperature only the former contributes due to the process of spontaneous phonon emission.
As we just mentioned, to forego the sum over states, we will make use of Dalgarno and Lewis' formulation of perturbation theory. In order to evaluate $\Delta E_{1}$ we introduce two operators $F_{1 \pm}$ which obey to the relations

$$
\left(\left[F_{1 \pm}, H_{0}\right] \pm \hbar \omega_{\mathbf{q}} F_{1 \pm}\right)|1 s\rangle=e^{\mp i \mathbf{q} \cdot \mathbf{r} m_{e} / M}|1 s\rangle .
$$

Now, we apply these to Eq. (7), remove the sum over states and introduce three complete sets of plane waves. Doing so, and taking advantage of the orthogonality relation between plane waves, one finds the following expression for $\Delta E_{1}$ :

$$
\begin{aligned}
\Delta E_{1}= & -\frac{2 \mu}{\hbar^{2}} \frac{U^{2}}{A} \sum_{\mathbf{q}} \sum_{\mathbf{k}} \frac{1}{q}\left[n_{p h}(\mathbf{q}, T)+1\right] \frac{\langle 1 s \mid \mathbf{k}\rangle\langle\mathbf{k} \mid 1 s\rangle}{q^{2}\left(\frac{m_{e}}{M}\right)^{2}+2 \mathbf{k} \cdot \mathbf{q} \frac{m_{e}}{M}+2 \mu \omega_{\mathbf{q}} / \hbar} \\
& -\frac{2 \mu}{\hbar^{2}} \frac{U^{2}}{A} \sum_{\mathbf{q}} \sum_{\mathbf{k}} \frac{1}{q} n_{p h}(\mathbf{q}, T) \frac{\langle 1 s \mid \mathbf{k}\rangle\langle\mathbf{k} \mid 1 s\rangle}{q^{2}\left(\frac{m_{e}}{M}\right)^{2}+2 \mathbf{k} \cdot \mathbf{q} \frac{m_{e}}{M}-2 \mu \omega_{\mathbf{q}} / \hbar} .
\end{aligned}
$$

Comparing Eqs. (7) and (10), we note that with the approach of Dalgarno and Lewis the problem of computing $\Delta E_{1}$ was drastically modified. While in Eq. (7) the knowledge of all the excitonic states was required, in (10) only the Fourier transform of the $1 s$ state wave function is needed. Moreover, the complexity of the calculations was greatly reduced, since now we need only compute two sums over the momenta $\mathbf{q}$ and $\mathbf{k}$ while previously, the computation of an infinite number of matrix elements was required. Finally we note that in Eq. (10) the operators $F_{1 \pm}$ are absent, since we do not need them explicitly, but rather the expression for their matrix element between plane waves, which can be computed from Eq. (9). In order to progress analytically, we follow a variational path to describe the wave function of the $1 \mathrm{~s}$ excitonic state. Our variational ansatz, inspired by the Hydrogen atom, reads [14

$$
\psi_{1 s}(r)=a \sqrt{\frac{2}{\pi}} e^{-r / a}, \quad \psi_{1 s}(\mathbf{k})=\frac{2 a \sqrt{2 \pi}}{\left(1+a^{2} k^{2}\right)^{3 / 2}},
$$

where $a$ is a variational parameter determined from the minimization of the expectation value of $H_{0}$, and can be roughly interpreted as the excitonic Bohr radius. This choice of the trial wave function produces wave functions in good agreement with the ones found using exact numerical methods. A more sophisticated ansatz combining two exponential functions [15] could also be used. This option would produce results in perfect agreement with the numerical ones, with the cost of more involved calculations, without a simple analytical solution. In any case, the choice of one of the ansätze over the other should produce only minimal changes in the final result. To continue with the calculation of $\Delta E_{1}$ the sums over $\mathbf{q}$ and $\mathbf{k}$ must be converted into two-dimensional integrals. From this point onward we explicitly consider that $\omega_{\mathbf{q}}=\omega_{\mathrm{LO}}$; as a consequence the terms $n_{p h}(\mathbf{q}, T)$ become momentum independent and can be taken out of the integrals. Writing the integrals in polar coordinates, one finds the following angular integral

$$
\int_{0}^{2 \pi} \frac{d \theta}{\sigma_{ \pm}+k \cos \theta}= \begin{cases}\operatorname{sign} \sigma_{ \pm} \frac{2 \pi}{\sqrt{\sigma_{ \pm}^{2}-k^{2}}}, & \left|\sigma_{ \pm}\right|>1 \\ 0, & \left|\sigma_{ \pm}\right|<1\end{cases}
$$

where $\sigma_{ \pm}=\left(q^{2} \pm 2 \mu \omega_{\mathrm{LO}}\right) / 2 q$. When $\left|\sigma_{ \pm}\right|<1$ the principal value of the integral should be considered. We now note that for the second term in Eq. (10), the term associated with absorption of phonons, $\sigma_{-}= \pm 1$ depending on the value of $q$. The same does not apply to the other contribution, where $\sigma_{+}>0 \forall q \geq 0$. As a consequence of the two possible signs that originate from the angular integration, when the integrals over $d k$ and $d q$ are computed the phonon absorption contribution from $\Delta E_{1}$ (and $\Delta E_{2}$ after the roles of $m_{e}$ and $m_{h}$ are switched) vanishes. Computing the remaining integrals, we find

$$
\begin{gathered}
\Delta E_{1}=-\frac{4 m_{h}}{\pi \hbar^{2}} U^{2} a^{2}\left[n_{p h}\left(\omega_{\mathrm{LO}}, T\right)+1\right] \times \\
{\left[\frac{\pi\left(\chi_{+}^{2}-1\right)\left(4 \chi_{+}^{2}+3\right)}{32 a^{3} \beta^{2} \chi_{+}^{3}}+f_{+}\right],}
\end{gathered}
$$

with $\chi_{+}=1+a^{2} \beta^{2}, \beta^{2}=2 \mu \omega_{\mathrm{LO}} / \hbar$ and

$$
f_{+}=\int_{0}^{\infty} \frac{1}{16 q} \frac{3 \operatorname{arcsinh}\left[a \zeta_{+}(q)\right]}{a\left[1+a^{2} \zeta_{+}^{2}(q)\right]^{5 / 2}} d q \approx \frac{3}{8 \pi a},
$$

where $\zeta_{+}(q)=\left(q^{2}+\beta^{2}\right) / 2 q$. The value of $f_{+}$is roughly less that one half of the the term with which it is summed. As we have already mentioned, the contribution $\Delta E_{2}$ is obtained from $\Delta E_{1}$ by substituting $m_{h}$ with $m_{e}$. 
Now that $\Delta E_{1}$ and $\Delta E_{2}$ were computed, we turn our focus to the contributions $\Delta E_{3}$ and $\Delta E_{4}$. The process to compute these terms is very much alike the one described for the other two. Similarly to before, we start by defining the operators $F_{3 \pm}$ which obey to the relation
$\left(\left[F_{3 \pm}, H\right] \pm \hbar \omega_{\mathbf{q}} F_{3 \pm}\right)|1 s\rangle=e^{-i \mathbf{q} \cdot\left(m_{e / h} / M\right) \mathbf{r}}|1 s\rangle$. The introduction of these operators allows us to remove the sum over states. After the plane wave basis have been introduced, and their orthogonality relations employed, we arrive at the following expression:

$$
\begin{aligned}
\Delta E_{3} & =-\frac{2 \mu}{\hbar^{2}} \frac{U^{2}}{A} \sum_{\mathbf{q}} \sum_{\mathbf{k}} \frac{1}{q}\left\{\left[n_{p h}(\mathbf{q}, T)+1\right] \frac{\langle 1 s \mid \mathbf{k}\rangle\langle\mathbf{k}+\mathbf{q} \mid 1 s\rangle}{q^{2} \frac{\left[2 m_{e}+m_{h}\right] m_{h}}{M^{2}}+2 \mathbf{k} \cdot \mathbf{q} \frac{m_{h}}{M}-2 \mu \omega_{\mathbf{q}} / \hbar}\right. \\
& \left.+n_{p h}(\mathbf{q}, T) \frac{\langle 1 s \mid \mathbf{k}\rangle\langle\mathbf{k}+\mathbf{q} \mid 1 s\rangle}{q^{2} \frac{\left[2 m_{h}+m_{e}\right] m_{e}}{M^{2}}+2 \mathbf{k} \cdot \mathbf{q} \frac{m_{e}}{M}+2 \mu \omega_{\mathbf{q}} / \hbar}\right\} .
\end{aligned}
$$

Comparing this result with Eq. 10, we note that in the present case the Fourier transforms of the $1 s$ wave function are evaluated at different momenta. This modification significantly increases the complexity of the integrals that must be computed, preventing the existence of a simple analytical solution. The angular integrals can, however, be computed analytically, yielding:

$$
\begin{aligned}
& \int_{0}^{2 \pi} \frac{d \theta}{\left[\xi_{ \pm}+\cos \theta\right][\delta+\cos \theta]^{3 / 2}}= \\
= & -4 \frac{\left(1+\xi_{ \pm}\right) \mathbf{E}\left(\frac{2}{1+\delta}\right)-(\delta-1) \mathbf{\Pi}\left(\frac{2}{1+\xi_{ \pm}} \mid \frac{2}{1+\delta}\right)}{\left(1+\xi_{ \pm}\right) \sqrt{\delta+1}(\delta-1)\left(\delta-\xi_{ \pm}\right)},
\end{aligned}
$$

where $\mathbf{E}(x)$ and $\boldsymbol{\Pi}(x \mid y)$ are elliptic integrals of the second and third kind, respectively, $\delta=\left(a^{-2}+k^{2}+q^{2}\right) / 2 k q>$ 1 and $\xi_{ \pm}=\left(q^{2} \frac{\left[2 m_{h / e}+m_{e / h}\right]}{M} \pm \frac{2 m_{h / e} \omega_{\mathrm{LO}}}{\hbar}\right) / 2 k q$. This solution is valid for both $\left|\xi_{ \pm}\right|>1$ and $\left|\xi_{ \pm}\right|<1$. For the latter case, the principal value of the integral should be considered. The remaining integrals over $d k$ and $d q$ do not yield analytical solutions, and as a consequence must be evaluated numerically, taking the principal value of the integral when necessary. When doing so, one must proceed carefully, starting by determining the points where poles and branch cuts appear. These points correspond to the ones where the the conditions $\xi_{-}=-1$ and $\xi_{ \pm}=1$ are satisfied; in addition care must be exercised when $\delta-\xi_{ \pm}$.

Up to this point we have described and given equations that characterize the exciton-phonon interaction. As an application of the results so far derived we will produce a theoretical description of the experimental data presented in Ref. [6], where the shift of the $1 s$ excitonic resonance was measured as a function of the temperature. To accurately describe this effect we must consider something so far ignored. As the temperature increases two distinct effects take place. On the one hand, the exciton-phonon interaction, dominated by LO phonons, will modify the exciton binding energy, shifting the excitonic peak; this is the effect we have theoretically de- scribed. On the other hand, the band gap decreases as the temperature increases, also contributing to the excitonic resonance shift. To describe this effect the Varshni empirical model [16] is commonly used, however we choose to consider the vibronic model of Huang and Rhys [17, which takes into account the effect of acoustic phonons:

$$
E_{g}(T)=E_{g}(0)-S\left\langle\hbar \omega_{\mathrm{A}}\right\rangle\left[\operatorname{coth} \frac{\left\langle\hbar \omega_{\mathrm{A}}\right\rangle}{2 k_{B} T}-1\right]
$$

where $E_{g}(T)$ is the band gap magnitude at a temperature $T,\left\langle\hbar \omega_{\mathrm{A}}\right\rangle$ is the mean energy of the acoustic phonons (about $10 \mathrm{meV}$ [18]), $k_{B}$ is the Boltzmann constant, and $S$ is a fitting parameter of the order of 1 , describing the electron-acoustic-phonon coupling. The expression for the $1 s$ resonance position as a function of $T$, which we label as $E_{X}(T)$, relatively to its position at $T=0 \mathrm{~K}$, reads:

$$
\begin{aligned}
E_{X}(T)-E_{X}(0) & =-S\left\langle\hbar \omega_{\mathrm{A}}\right\rangle\left[\operatorname{coth} \frac{\left\langle\hbar \omega_{\mathrm{A}}\right\rangle}{2 k_{B} T}-1\right] \\
& +E_{B}(T)-E_{B}(0),
\end{aligned}
$$

where $E_{B}(T)<0$ is the $1 s$ state binding energy at a temperature $T$. This quantity can be obtained using [9]

$$
E_{B}(T)=E_{a b \text { initio }}+\Delta E(T)+2 \alpha \hbar \omega_{\mathrm{LO}},
$$

where $E_{a b \text { initio }}$ is the $1 s$ binding energy of the unperturbed system, that is, when no phonons are present, $\Delta E(T)=\Delta E_{1}+\Delta E_{2}+\Delta E_{3}+\Delta E_{4}$ and $2 \alpha \hbar \omega_{\mathrm{LO}}$ is the sum of the free electron and hole polarons (where we assumed that $\alpha$ is approximately the same for electrons and holes). The value of $E_{a b \text { initio }}$ can be found numerically or with semi-analytical methods, however, since this is a temperature independent value, it vanishes from $E_{X}(T)-E_{X}(0)$. Using Eq. 19 we performed a fit to the experimental data of Ref. [6]. The comparison between our theoretical description and the experimental results is depicted in Figure 1, the parameters are presented in Table I. Inspecting Figure 1 we observe an excellent agreement between our theoretical description 


\begin{tabular}{ccccccccc}
\hline$\kappa$ & $r_{0}$ & $m_{e}$ & $m_{h}$ & $a$ & $\hbar \omega_{\mathrm{LO}}$ & $\alpha$ & $\left\langle\hbar \omega_{\mathrm{A}}\right\rangle$ & $S$ \\
\hline \hline 2.4 & 37.9 & 0.35 & 0.26 & 15 & 43 & 2 & 11 & 1.32 \\
\hline
\end{tabular}

Table I. Parameters used to model the problem, and the fitting parameters. The distances are given in $\AA$, the masses in units of the electron bare mass, and the energies in $\mathrm{meV}$. The value of $\kappa$ corresponds to the mean dielectric constant of vacuum and $\mathrm{SiO}_{2}$. The value of $r_{0}$ was taken from Ref. [19, and the values of $m_{e}$ and $m_{h}$ from Ref. [20. The value of $a$ was obtained from the minimization of $H_{0}$ using the parameters just mentioned. The value for the LO phonons energy was taken from Ref. [18. The values of $\alpha,\left\langle\hbar \omega_{\mathrm{A}}\right\rangle$ and $S$ were obtained from the fit of the theoretical model to the data of Ref. 6.

and the experimental data-points. At room temperature the gap renormalization is responsible for a shift of approximately $65 \mathrm{meV}$ while the polaron shift contributes with approximately $15 \mathrm{meV}$, in rough agreement with the values found in Ref. [7]. Analyzing the content of Table II we note that the fitting parameters, $\alpha,\left\langle\hbar \omega_{\mathrm{A}}\right\rangle$ and $S$ are in agreement with previous results found in the literature. The value of $\alpha$ lies inside the interval between 0 and 5 indicated in Ref. [11. The value of $\left\langle\hbar \omega_{\mathrm{A}}\right\rangle$ matches the one obtained in Ref. [18 and used in Ref. [21, where $\mathrm{MoSe}_{2}$ was studied. In Ref. [18] a value of $S=1.93$ was found for $\mathrm{MoSe}_{2}$. Using the fact that this parameter, which characterizes the coupling to phonons, should be proportional to the square root of the effective masses, and noting that the effective masses in $\mathrm{MoSe}_{2}$ differ from those in $\mathrm{WS}_{2}$ approximately by a factor of $0.7^{2}$ [20] we can estimate that the value of $S$ in $\mathrm{WS}_{2}$ should be around 1.3, in total agreement with the value we found.

In summary, using second order perturbation theory we have successfully described the effect of temperature on aset of experimental data-points on the position of the fundamental absorption line peak of the 1s excitonic transition (corresponding to the more tightly bound exciton) in $\mathrm{WS}_{2}$ [6]. The experiment shows a red shift of the absorption line when the temperature increases. We were able to describe, in quantitative terms, the observed shift considering two different effects: the polaron shift and the renormalization of the single particle gap with temperature. Both effects were shown to produce a sizable effect to the overall red shift, and should be accounted for in theoretical descriptions of this phenomenom. We stress that both effects are due to different set of phonons: the longitudinal optical phonons in the intrinsic red shift of the absorption line and the acoustic phonons in the modification of the single particle gap. A follow up of this work will focus on the calculation of line shape of the absorption peak as a function of temperature which requires solving the Bethe-Salpeter equation in the presence of the phonon's field.

N.M.R.P acknowledges support by the Portuguese Foundation for Science and Technology (FCT) in the framework of the Strategic Funding UIDB/04650/2020.

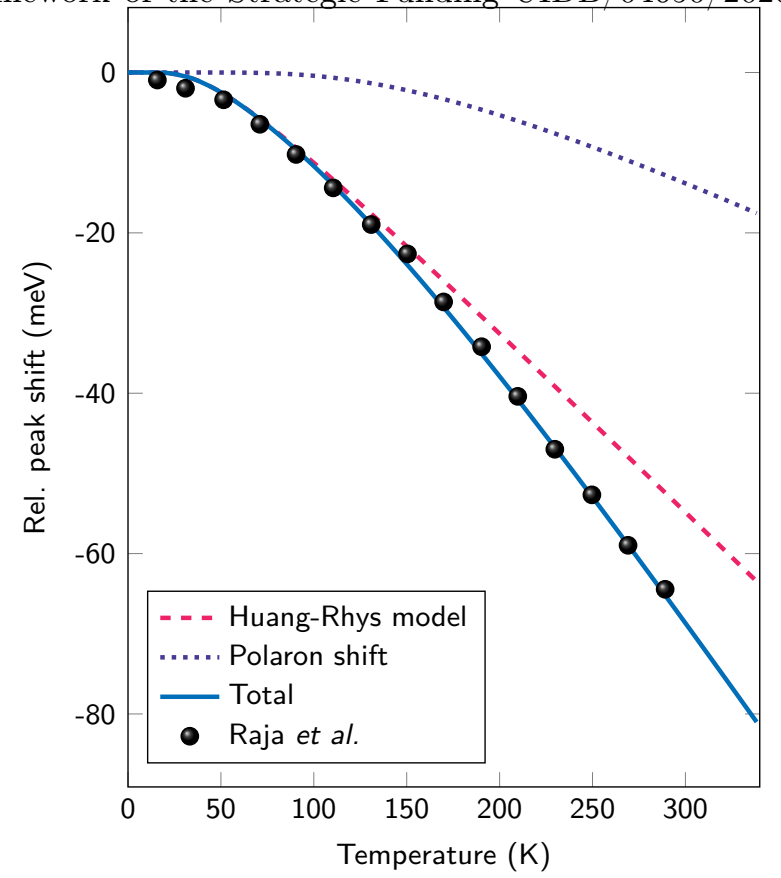

Figure 1. Comparison between the fit obtained with Eq. 19 and the experimental data of Ref. [6. An excelent agreement between the theoretical description and the experimental points is evident. Also depicted are the isolated contributions of the polaron shift, and the gap renormalization described with the Huang-Rhys model. The value of the fitting parameters is given in Table $\mathrm{I}$.

J.C.G.H. acknowledges the Center of Physics for a grant funded by the UIDB/04650/2020 strategic project. N.M.R.P. acknowledges support from the European Commission through the project "Graphene-Driven Revolutions in ICT and Beyond" (Ref. No. 881603, CORE 3), COMPETE 2020, PORTUGAL 2020, FEDER and the FCT through projects POCI-01-0145-FEDER028114, PTDC/NAN-OPT/29265/2017
[1] Q. H. Wang, K. Kalantar-Zadeh, A. Kis, J. N. Coleman, and M. S. Strano, "Electronics and optoelectronics of two-dimensional transition metal dichalcogenides," Nature nanotechnology, vol. 7, no. 11, pp. 699-712, 2012.

[2] D. Li, C. Trovatello, S. Dal Conte, M. Nuß, G. Soavi, G. Wang, A. C. Ferrari, G. Cerullo, and T. Brixner,
"Exciton-phonon coupling strength in single-layer MoSe2 at room temperature," Nature Communications, vol. 12, no. 1, p. 954, Feb. 2021.

[3] P.-F. Li and Z.-W. Wang, "Optical absorption of fröhlich polaron in monolayer transition metal dichalcogenides," Journal of Applied Physics, vol. 123, no. 20, p. 204308, 
2018.

[4] Z.-W. Wang, W.-P. Li, Y. Xiao, R.-Z. Li, and Z.-Q. Li, "Influence of exciton-phonons coupling on the exciton binding energy in monolayer transition metal dichalcogenides," Applied Physics Letters, vol. 110, no. 23, p. 231603, 2017.

[5] S. Shree, M. Semina, C. Robert, B. Han, T. Amand, A. Balocchi, M. Manca, E. Courtade, X. Marie, T. Taniguchi et al., "Observation of exciton-phonon coupling in mose 2 monolayers," Physical Review B, vol. 98, no. 3, p. 035302, 2018.

[6] A. Raja, M. Selig, G. Berghauser, J. Yu, H. M. Hill, A. F. Rigosi, L. E. Brus, A. Knorr, T. F. Heinz, E. Malic et al., "Enhancement of exciton-phonon scattering from monolayer to bilayer ws2," Nano letters, vol. 18 , no. 10 , pp. 6135-6143, 2018.

[7] D. Christiansen, M. Selig, G. Berghäuser, R. Schmidt, I. Niehues, R. Schneider, A. Arora, S. M. de Vasconcellos, R. Bratschitsch, E. Malic et al., "Phonon sidebands in monolayer transition metal dichalcogenides," Physical review letters, vol. 119, no. 18, p. 187402, 2017.

[8] A. Dalgarno and J. T. Lewis, "The exact calculation of long-range forces between atoms by perturbation theory," Proceedings of the Royal Society of London. Series A. Mathematical and Physical Sciences, vol. 233, no. 1192, pp. 70-74, 1955.

[9] J. De Vooght and K. Bajaj, "Ground-state energy of a wannier exciton in a polar crystal," Physical Review B, vol. 7 , no. 4 , p. $1472,1973$.

[10] H. Fröhlich, "Electrons in lattice fields," Advances in Physics, vol. 3, no. 11, pp. 325-361, 1954.

[11] J. T. Devreese and F. M. Peeters, The physics of the twodimensional electron gas. Springer Science \& Business Media, 2012, vol. 157.

[12] L. Keldysh, "Coulomb interaction in thin semiconduc- tor and semimetal films," Sov. J. Exp. and Theor. Phys. Lett., vol. 29, p. 658, 1979.

[13] S. Rytova, "The screened potential of a point charge in a thin film," Moscow University Physics Bulletin, vol. 22, no. 30, 1967.

[14] M. F. M. Quintela and N. M. Peres, "A colloquium on the variational method applied to excitons in $2 \mathrm{~d}$ materials," The European Physical Journal B, vol. 93, no. 12, pp. 1-16, 2020.

[15] T. G. Pedersen, "Exciton stark shift and electroabsorption in monolayer transition-metal dichalcogenides," Physical Review B, vol. 94, no. 12, p. 125424, 2016.

[16] Y. P. Varshni, "Temperature dependence of the energy gap in semiconductors," physica, vol. 34, no. 1, pp. 149154, 1967.

[17] K. Huang and A. Rhys, Theory of light absorption and non-radiative transitions in F-centres. World Scientific, 2000.

[18] S. Tongay, J. Zhou, C. Ataca, K. Lo, T. S. Matthews, J. Li, J. C. Grossman, and J. Wu, "Thermally driven crossover from indirect toward direct bandgap in $2 \mathrm{~d}$ semiconductors: Mose2 versus mos2," Nano letters, vol. 12, no. 11, pp. 5576-5580, 2012.

[19] A. Chaves, R. Ribeiro, T. Frederico, and N. Peres, "Excitonic effects in the optical properties of $2 \mathrm{~d}$ materials: an equation of motion approach," 2D Materials, vol. 4, no. 2, p. 025086, 2017.

[20] A. Kormányos, G. Burkard, M. Gmitra, J. Fabian, V. Zólyomi, N. D. Drummond, and V. Fal'ko, "k · p theory for two-dimensional transition metal dichalcogenide semiconductors," 2D Materials, vol. 2, no. 2, p. 022001, 2015.

[21] B. K. Choi, M. Kim, K.-H. Jung, J. Kim, K.-S. Yu, and Y. J. Chang, "Temperature dependence of band gap in mose2 grown by molecular beam epitaxy," Nanoscale research letters, vol. 12, no. 1, pp. 1-7, 2017. 